\title{
Arquitectura monumental final (2500-2250 cal AC) en el yacimiento de Perdigões (Portugal). Aspectos formales y cronológicos a partir del estudio de Foso 2*
}

\author{
Late monumental architecture (2500-2250 cal BC) at Perdigões site (Portugal): form and \\ chronology based on the study on Ditch 2
}

\author{
Lara Milesia, José Enrique Márquez-Romerob, José Suárez Padilla ${ }^{\mathrm{b}}$ y José Luis Caro- \\ Herrero $^{b}$
}

\section{RESUMEN}

La investigación de los recintos de fosos de la Prehistoria de la península ibérica ha vivido un gran avance en las últimas dos décadas. Uno de los casos más importantes del sur peninsular es el conocido yacimiento arqueológico de Perdigões (Portugal). Presentamos aquí el estudio del denominado Foso 2, dando a conocer su morfología, la dinámica estratigráfica de su relleno y su cronología, para la que se cuenta con 12 dataciones radiocarbónicas inéditas. Los resultados de estas últimas han puesto de manifiesto que, junto al Foso 1, estos dos grandes recintos configuran una fase monumental tardía del yacimiento, acontecida entre el 2500 - 2250 cal AC. Por último, el estudio cronológico confirma la importancia que tuvo la presencia de un grupo de sepulcros tipo tholos más antiguos para el trazado de dichos fosos.

\begin{abstract}
Research on Iberian Prehistoric ditched enclosures has seen a significant progress in the last two decades. One of the best-known sites of Southern Iberia is Perdigões (Portugal). We present the study of the so-called Ditch 2, describing in detail its morphology, the stratigraphic sequence of its filling and its chronology, on the basis of 12 unpublished radiocarbon dates. Results confirm that this feature, together with Ditch 1, represents the last monumental phase of the site, dated in the third
\end{abstract}

quarter of the 3rd millennium. They also confirm the importance that a group of pre-existing tombs had in the layout of the ditches.

Palabras clave: Recinto de fosos; Perdigões; Portugal; Calcolítico; Prehistoria Reciente; Cronología.

Key words: Ditched enclosure; Perdigões; Portugal; Cooper Age; Late Prehistory; Chronology.

\section{INTRODUCCIÓN}

El yacimiento de Perdigões (Concelho de Reguengos de Monsaraz, Évora, Portugal) se extiende por más de 16 ha, emplazándose en la extremidad más occidental del valle del río Álamo, afluente del río Guadiana ( $38^{\circ} 26^{\prime} 26^{\prime \prime}$ N; $7^{\circ} 32^{\prime} 52^{\prime \prime}$ O). Se asienta sobre una base geológica de dioritos y gabros, integrada en la peniplanicie del Alentejo Central (Fig. 1).

En el momento actual se conocen en Perdigões trece fosos sensiblemente concéntricos excavados en el sustrato geológico (Márquez et al. 2011b; Valera et al. 2017); un área funeraria con varios sepulcros, algunos tipo tholos (Valera et al. 2000), los restos de un crómlech (Varela Gomes 1994: 327) y centenares de otras estructuras menores, en su mayoría negativas,

\footnotetext{
* Proyecto I+D Excelencia y Retos: "Arqueología y patrimonio en los recintos de fosos. Teledetección, caracterización y protección en yacimientos del suroeste de la Península Ibérica” (ref. HAR2014-53692-P). Ministerio de Economía y Competitividad. Investigador principal J. E. M-R.

a Universidad de Granada. Facultad de Filosofía y Letras. Campus de Cartuja s/n. 18071 Granada. Correo e.: lmilesi@ugr.es https://orcid.org/0000-0001-9002-015X

b Universidad de Málaga. Facultad de Filosofía y Letras. Campus de Teatinos s/n. 29010 Málaga. Correos e.: jemarquez@uma.es https://orcid.org/0000-0003-1861-8338; jsuarez@uma.es https://orcid.org/0000-0001-6795-8668; jlcaro@uma.es https://orcid.org/0000-0002-6587-0838

Recibido 18-V-2018; aceptado 12-IX-2018.
} 


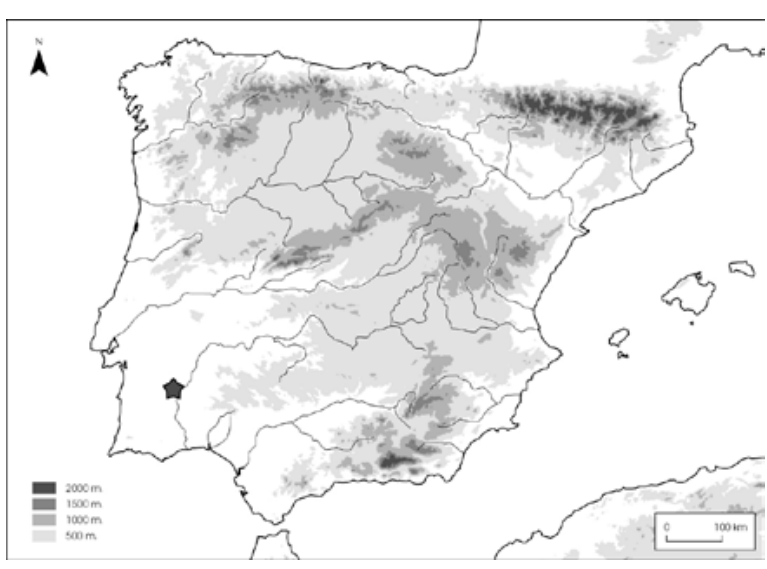

Fig. 1. Localización del yacimiento arqueológico de Perdigões (Portugal) en la península ibérica.

que se distribuyen por toda el área del yacimiento (Fig. 2).

Desde que se diera a conocer a mediados de los años 1980 sucesivas intervenciones arqueológicas de distinta naturaleza fueron caracterizando progresivamente el espacio (Varela Gomes 1994; Lago et al. 1998; Valera et al. 2000). A partir de 2006 se inició el denominado Programa Global de Investigação Arqueológica dos Perdigões (INARP), desarrollado por el Núcleo de Investigação Arqueológica (NIA) de ERA Arqueologia dentro del cual, y desde entonces, se vienen programando y coordinando todas las actuaciones arqueológicas en el yacimiento. Gracias a la investi- gación así desarrollada es uno de los recintos de fosos con mayor información arqueológica de la península ibérica como recientemente se ha apuntado (Valera 2017).

A partir del año 2008, la Universidad de Málaga se integró en dicho Programa Global con un proyecto propio (Márquez et al. 2008: 43). La zona de actuación elegida para los trabajos arqueológicos fue la entrada o punto de acceso denominada Puerta 1 incluida dentro del "Sector L" en la zona noreste de Perdigões. Las campañas arqueológicas se han sucedido desde 2008 hasta 2016 (Márquez et al. 2011a, 2011b; Suárez-Padilla et al. 2013, 2015). En 2016 se excavó el Foso 2, del que damos a conocer, de forma novedosa, su morfología general, la estratigrafía de su relleno y las características básicas de la cultura material recuperada. Además, se presenta una serie de 12 dataciones radiocarbónicas obtenidas en muestras de su relleno.

Con los nuevos datos disponibles aportados por dicha excavación y las dataciones disponibles, nuestro objetivo ha consistido en analizar y comprobar la relación existente entre los Fosos 1 y 2, entendidos como las dos posibles últimas grandes construcciones del yacimiento (Valera et al. 2014b), cuya amortización marcaría el fin de la construcción y uso de este tipo de arquitectura en el lugar. Otro objetivo es el de comprender la relación de ambos fosos con los sepulcros tipo tholos que aparecen localizados entre ellos, en un área que fue interpretada como una necrópolis (Valera et al. 2000: 88).

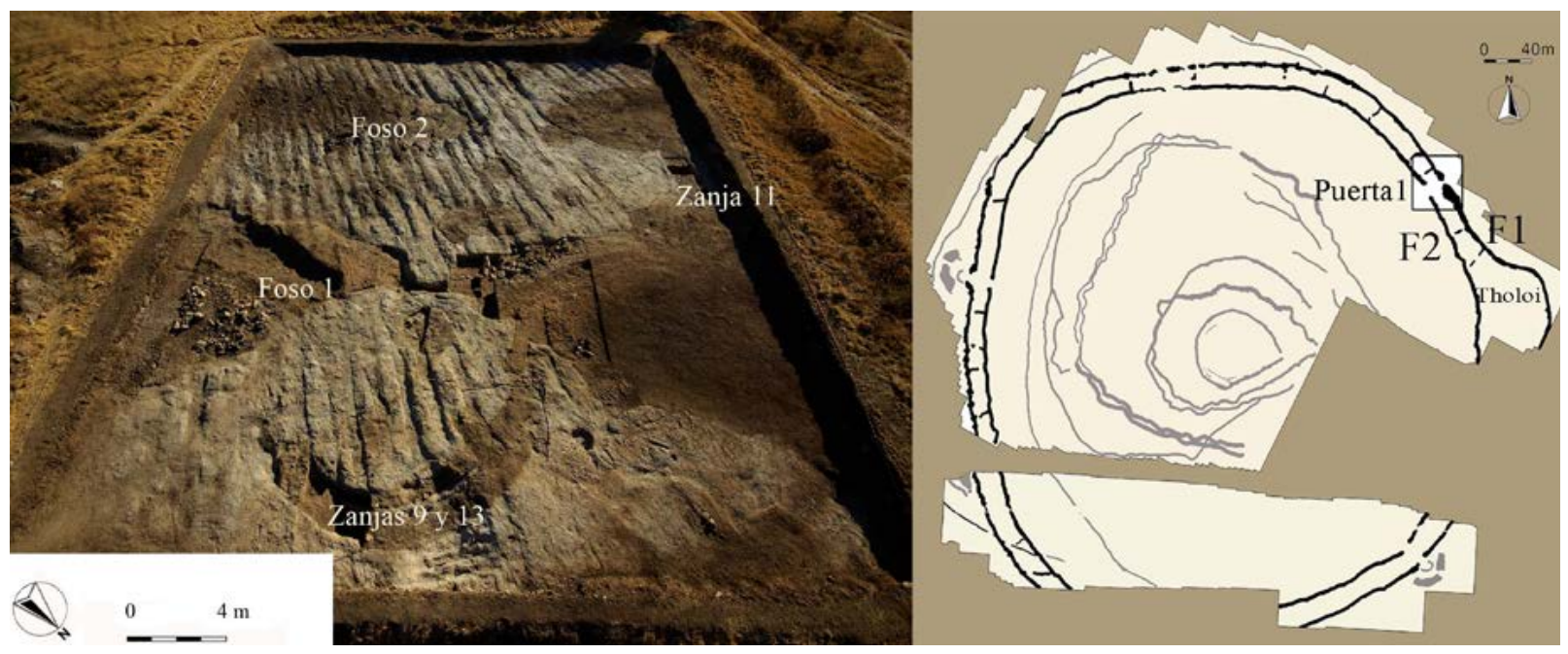

Fig. 2. Perdigões Corte L1: 1. Fotografía mostrando las numerosas estructuras excavadas y rellenadas en la Puerta 1. Destacan el Foso 1, el Foso 2 y las Zanjas 9, 11 y 13; 2. Planta del yacimiento con representación de fosos (F) y localización del corte (a partir de Márquez et al. 2011a: 160) (en color en la versión electrónica).

Trab. Prehist., 76, N. ${ }^{\circ}$ 1, enero-junio 2019, pp. 161-176, ISSN: 0082-5638

https://doi.org/10.3989/tp.2019.12232 


\section{MORFOLOGÍA, SECUENCIA ESTRATIGRÁFICA Y CULTURA MATERIAL DEL FOSO 2}

Los trabajos de excavación de la Universidad de Málaga en el yacimiento de Perdigões se han concentrado en el denominado corte L1, un área de excavación en extensión con una superficie de $30 \times 34 \mathrm{~m}$, localizada en el sector noreste del yacimiento (Fig. 2). Bajo un potente estrato superficial (unos 60-80 cm), resultante de remociones de tierra contemporáneas para el cambio de cultivo de la parcela, aparecieron numerosas estructuras arqueológicas que formaban parte del complejo entramado conocido como Puerta 1 (Fig. 2). Casi todas las evidencias estratigráficas halladas en este sector son estructuras en negativo, en su mayoría, prehistóricas. Entre ellas está una parte del trazado del denominado Foso 2, uno de los grandes recintos del yacimiento, con aspecto de gran circunferencia (diámetro de unos $430 \mathrm{~m}$ ), perímetro aproximado de 1380 $\mathrm{m}$ y varios puntos de acceso. Esta gran estructura negativa se estudió mediante una sección transversal de 6 por $3 \mathrm{~m}$ (Fig. 3).

Durante las excavaciones se constató que el Foso 2 cortaba una estructura negativa preexistente, denominada Zanja 14. Tenía 1,38 m de profundidad, pare-

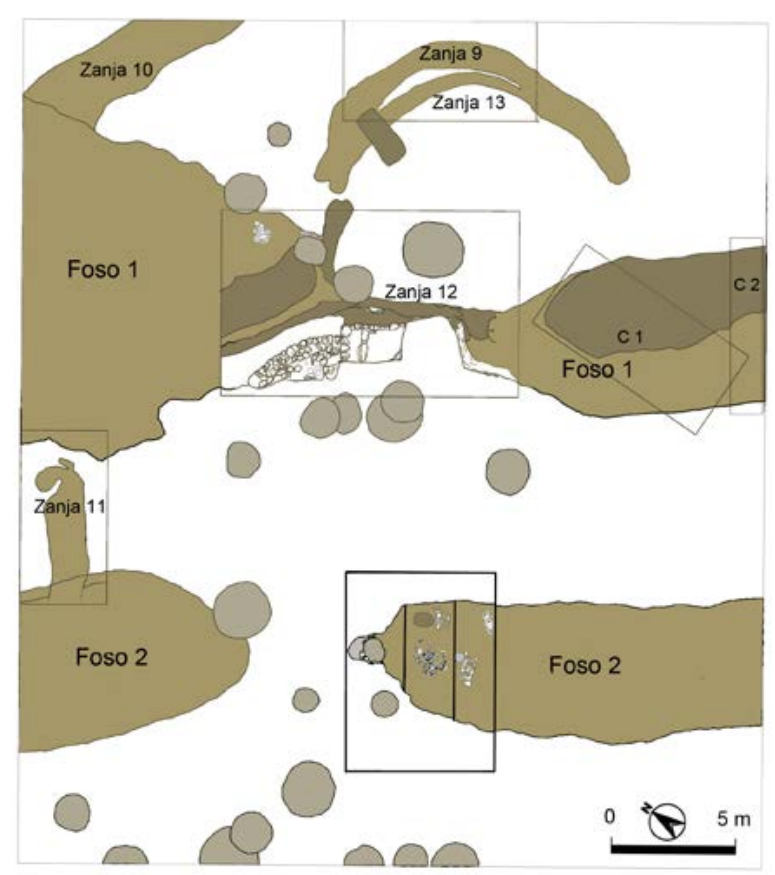

Fig. 3. Perdigões: planta del corte L1 (Puerta 1). Los recuadros señalan los cortes de excavación principales. En Foso 1 se indican C 1: corte 1, C 2: corte 2. Cada estructura muestra su numeración (en color en la versión electrónica). des oblicuas y sección en forma de cubeta con el fondo ligeramente aplanado. El primer estrato de colmatación de esta zanja (UE 629) presenta matriz arenosa con algunas piedras de tamaño mediano, escasos fragmentos cerámicos y una pequeña esquirla de hueso. Sobre este nivel se alternan finos estratos con matriz arenosa y apenas con material arqueológico, con otros cuyo componente mayoritario es el sustrato geológico descompuesto ${ }^{1}$ (Figs. 4 y 5 ).

La anchura máxima del Foso 2, documentada en este sector, es de 4,60 m y la profundidad de 2,09 m. La sección tiene forma de "V", aunque su remate final es algo redondeado. Además el trazado de sus respectivas paredes diverge ligeramente.

Los estratos de colmatación de Foso 2 podrían agruparse dentro de dos dinámicas de relleno diferentes, y a un momento posterior de amortización, en el que también se realizan fosas sobre el relleno (Fig. 6). La primera dinámica corresponde a los estratos más profundos, resultado de vertidos alternantes (Unidades Estratigráficas (UU.EE) 608, 607, 606, 605, 604, 603, $602,598,591)$ desde los extremos exteriores del foso que generan un encabalgamiento de los depósitos. Entre algunos de estos niveles de colmatación a veces se intercalaron finas capas de color blanquecino y matriz semejante al sustrato geológico del lugar. Tienen entre 2 y $7 \mathrm{~cm}$ de potencia y se caracterizan por la ausencia de material arqueológico (UU.EE 600, 599, 589). Se interpretan como episodios de aportes coluviales. Su naturaleza y buzamiento manifiestan su caída desde el borde interior del foso. Ello es una evidencia indirecta de la previsible presencia original de un bank, conformado por el propio sedimento geológico resultante de la excavación original de la gran estructura negativa.

Sobre estos rellenos, y hasta en dos ocasiones, se practicaron pequeñas fosas sensiblemente orientadas con el eje del foso, cuyo contenido presenta cierta concentración de material arqueológico, que contrasta con lo observado en los estratos a los que cortan (UU. EE 604 y 605).

\footnotetext{
1 Para conocer en profundidad la naturaleza de los depósitos se está desarrollando un estudio microestratigráfico de alta resolución de los rellenos, como parte de una línea de investigación del Proyecto $\mathrm{I}+\mathrm{D}$ Excelencia y Retos: "Arqueología y patrimonio en los recintos de fosos. Teledetección, caracterización y protección en yacimientos del suroeste de la Península Ibérica" (ref. HAR2014-53692-P). Ministerio de Economía y Competitividad. Esta línea la encabeza Lara Milesi (Universidad de Granada), en colaboración con Mario Gutiérrez (Universidad de Granada), Carlos Duarte (Universidad de Cantabria) y Paul Goldberg (Universidad de Boston). A modo de avance, los análisis de láminas delgadas confirman la existencia de episodios de origen natural y antrópico en los procesos de colmatación de las estructuras. Los primeros coinciden con los estratos con un alto contenido en 'geológico descompuesto' reconocidos en campo.
} 


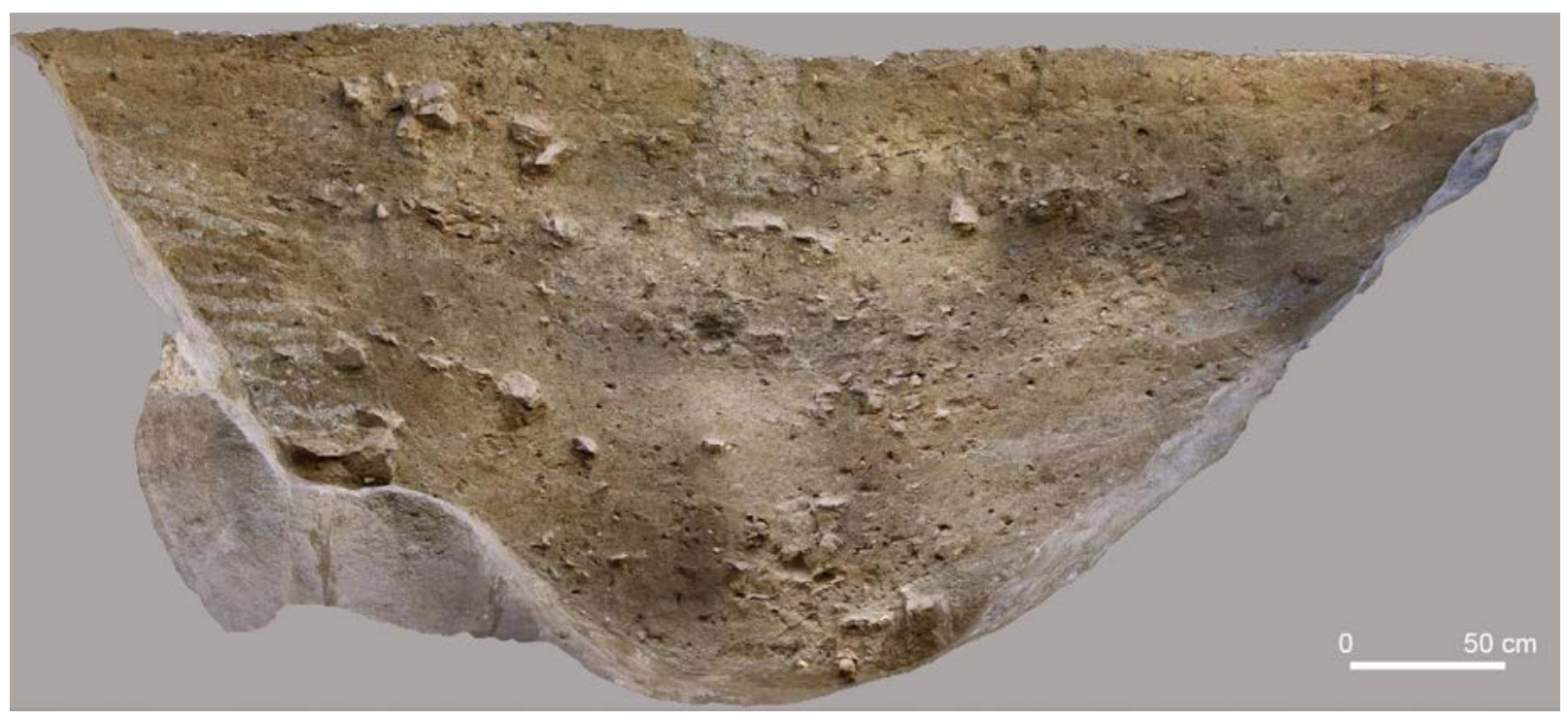

Fig. 4. Modelo fotogramétrico 3D del perfil de Foso 2. Se aprecia relleno de Zanja 14 y estratigrafía de Foso 2. A partir de colección de fotos de alta resolución y montaje con software Argisoft Photoscan (en color en la versión electrónica).

La segunda dinámica de relleno se conforma por niveles con una mayor tendencia a la horizontalidad, y menor potencia (UU.EE 596, 587, 588, 585, 583, $582,581,578,569,567,547)$, que suponen la amortización definitiva de la estructura. En ellos no es extraña la presencia de industria lítica, fragmentos cerámicos y restos faunísticos.

Como última fase de actividad prehistórica, se documentan actuaciones que ponen de manifiesto la continuidad del uso de este sector del yacimiento después del cierre de la estructura. Entre ellas, destacan una fosa asociada posiblemente a la inserción de un elemento vertical que solo presentaba el sustrato geológico descompuesto como relleno (UE 631) y un retazo de otra, de tendencia circular y poca profundidad, con revestimiento perimetral de obra de mampostería (UE 545).

Por último, una estructura negativa de época bajomedieval con aspecto cilíndrico, tipo pozo (F192), afecta parte de los rellenos de Foso 2 (Fig. 7).

La cultura material en este sector de Foso 2 consta de numerosos restos cerámicos: 5.492 fragmentos de cuerpos y 1.010 bordes, de los que 943 han podido ser caracterizados siguiendo la tipología vascular descrita para el propio yacimiento (Lago et al. 1998: 83- 85).

La pasta suele ser compacta, las superficies aparecen alisadas y raramente bruñidas y, por lo general, con desgrasantes abundantes con características petrológicas que, a nivel macroscópico, resultarían compatibles con la geología local. La mayoría de las formas son abiertas (tipos 1 y 2) y corresponden a grandes platos, pocos profundos (tipo 1: 48,99\%) y fuentes de borde engrosado (tipo 2: 24,71\%). Les siguen los cuencos semiesféricos profundos (forma 4: 14,63\%) y los recipientes de cuerpo esférico de menor tamaño (forma 5: 7,95\%). Finalmente, aparecen en baja proporción algunos fragmentos de tazas carenadas, cuencos de paredes rectas y base aplanada, recipientes globulares, vasos de cuerpo superior troncocónico, cuencos de carena media-baja y vasos de paredes rectas (tipos 3, 6, 7, 8, 10 y 13 respectivamente, que representan en conjunto el 3,71\% del total).

En los niveles de relleno con mayor concentración de materiales arqueológicos, aumenta la variedad de formas cerámicas, algo que no ocurre en el resto de los estratos. De este modo, se distinguen claras pulsiones que podrían indicar momentos de mayor actividad en el relleno.

Sólo se han localizado dos piezas completas: un vaso en miniatura (tipo 15) que procede de uno de los estratos más profundos y un vaso globular (tipo 7), que apareció en el interior del retazo de la fosa con pared de mampostería construida sobre los rellenos del Foso 2 (UE 541 en Fig. 6). La presencia de cerámica decorada es insignificante, destacando la ausencia de campaniforme.

Otros hallazgos fueron 15 fragmentos de pesas de telar de tendencia rectangular, 29 de "cuernecillos" de diferentes dimensiones, así como otro de "idolillo ginemorfo", con base plana, de unos $4,5 \mathrm{~cm}$ de ancho y dos perforaciones (Fig. 8: pieza 2573).

Los materiales líticos se reducen a 3 lascas irregulares y 5 puntas de flecha realizadas en sílex, con base cóncava y talla bifacial, repartidas en diferentes unida- 

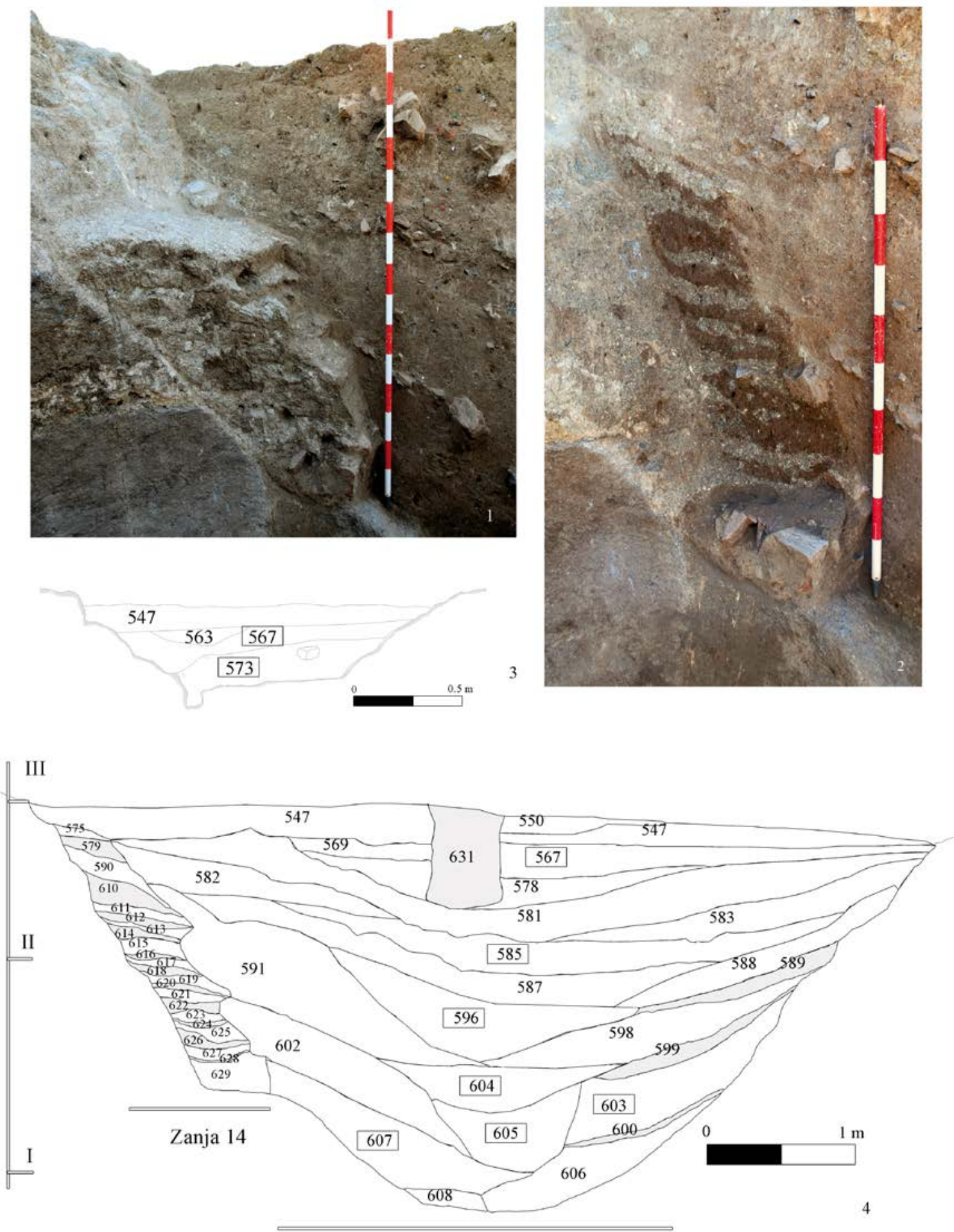

Foso 2

Fig. 5. Perdigões: Zanja 14: 1. relleno, 2. estratigrafía; Foso 2: 3. perfil oeste con UUEE dataciones radiocarbónicas; 4. Perfil este principal. Se recuadran las unidades estratigráficas que se han datado. I, II y III señalan los niveles en los que se evidencia el cambio de dinámica de relleno (1 y 2 en color en la versión electrónica). 


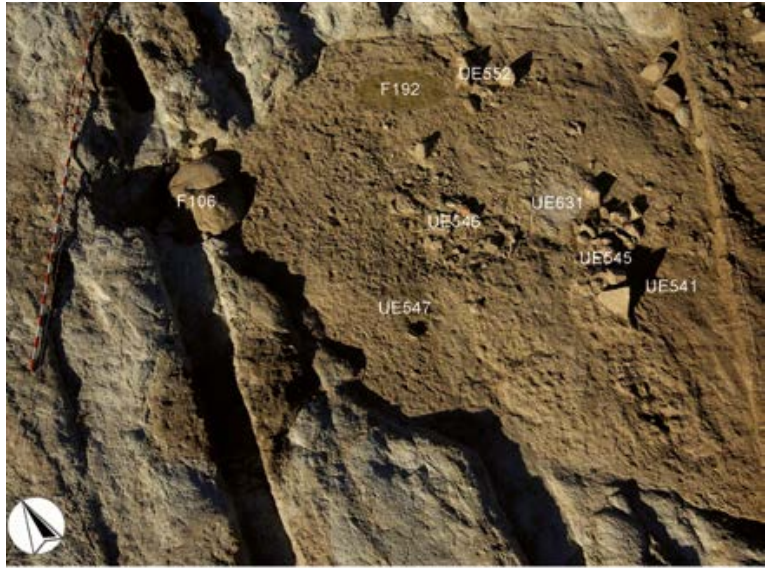

Fig. 6. Foso 2 de Perdigões. Unidades estratigráficas superficiales que evidencian la utilización del espacio una vez amortizado el foso. Destacan la UE 631, como relleno de fosa para la posible inserción de un elemento vertical y la UE 545 como base de una estructura de mampostería en positivo. Las UU.EE 546 y 552 son rellenos de fosas que han afectado a la UE547 que se extendía en toda la planta. F192 corresponde a la fosa bajomedieval (en color en la versión electrónica). des estratigráficas. Sin embargo, siete de las 10 manos de molino halladas se localizan en los estratos formados una vez amortizado el foso. Los tres restantes corresponden a niveles superiores. También hay cantos de origen aluvial de procedencia alóctona, que suelen aparecer con evidencias de fracturas intencionadas.

Los restos óseos faunísticos son abundantes durante toda la secuencia, y están en fase de estudio. También se documentó un hueso humano: corresponde a dos tercios de un húmero derecho de un individuo adulto y de sexo indeterminado ${ }^{2}$ en la UE 602.

\section{CRONOLOGÍA ABSOLUTA DE FOSO 2}

El estudio cronológico de Foso 2 consideró la complejidad del contexto arqueológico y las posibilidades de muestreo. La falta de elementos articulados en los

\footnotetext{
${ }^{2}$ La arqueozoóloga Cláudia Costa ha estudiado la fauna e identificado las muestras datadas con C14. Lucy Shaw Evangelista caracterizó el resto humano.
}

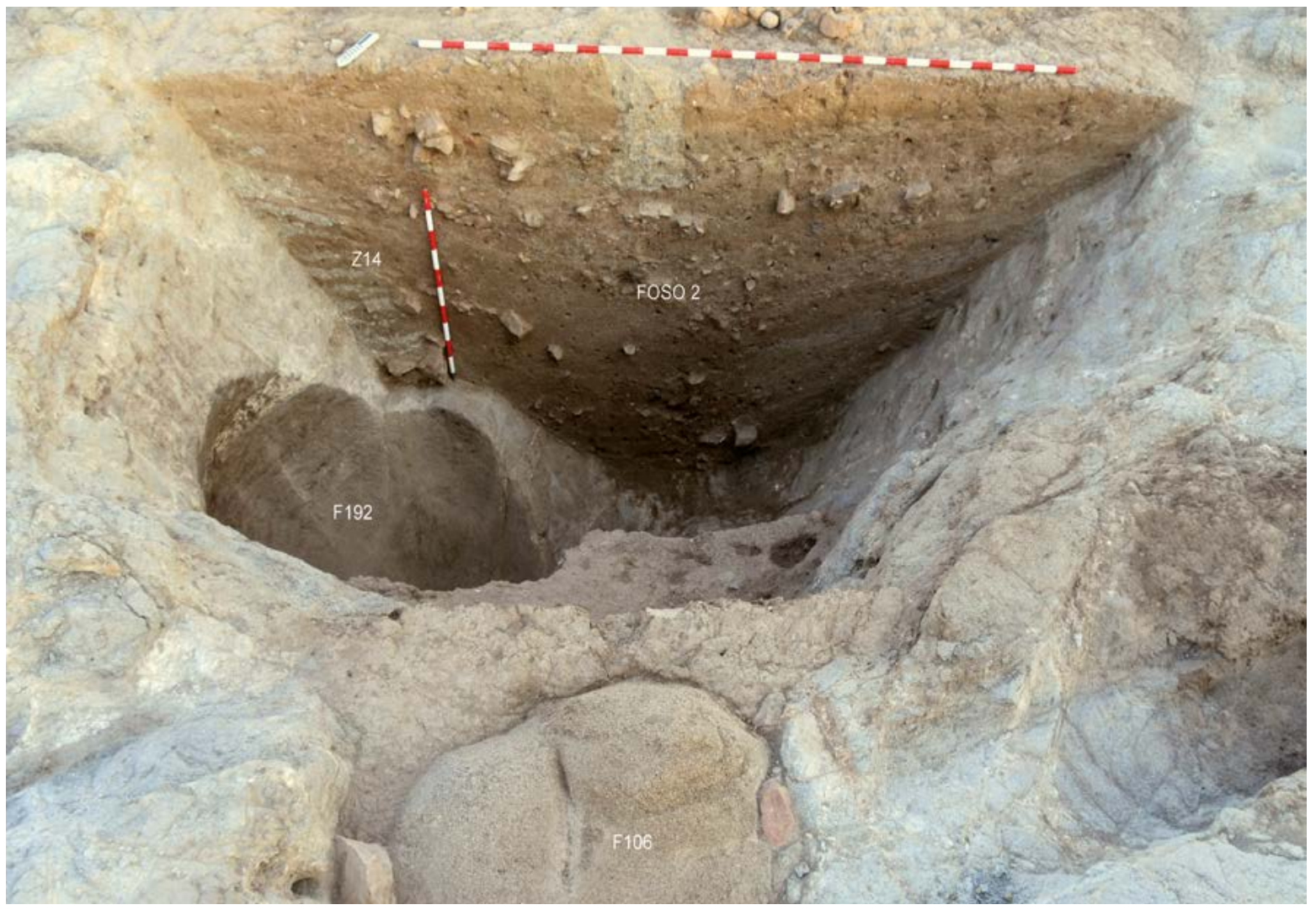

Fig. 7. Perfil este del Foso 2 de Perdigões. Se aprecian también la estratigrafía de la Zanja 14 y la Fosa 192 bajomedieval (en color en la versión electrónica).

Trab. Prehist., 76, N. ${ }^{\circ}$ 1, enero-junio 2019, pp. 161-176, ISSN: 0082-5638

https://doi.org/10.3989/tp.2019.12232 


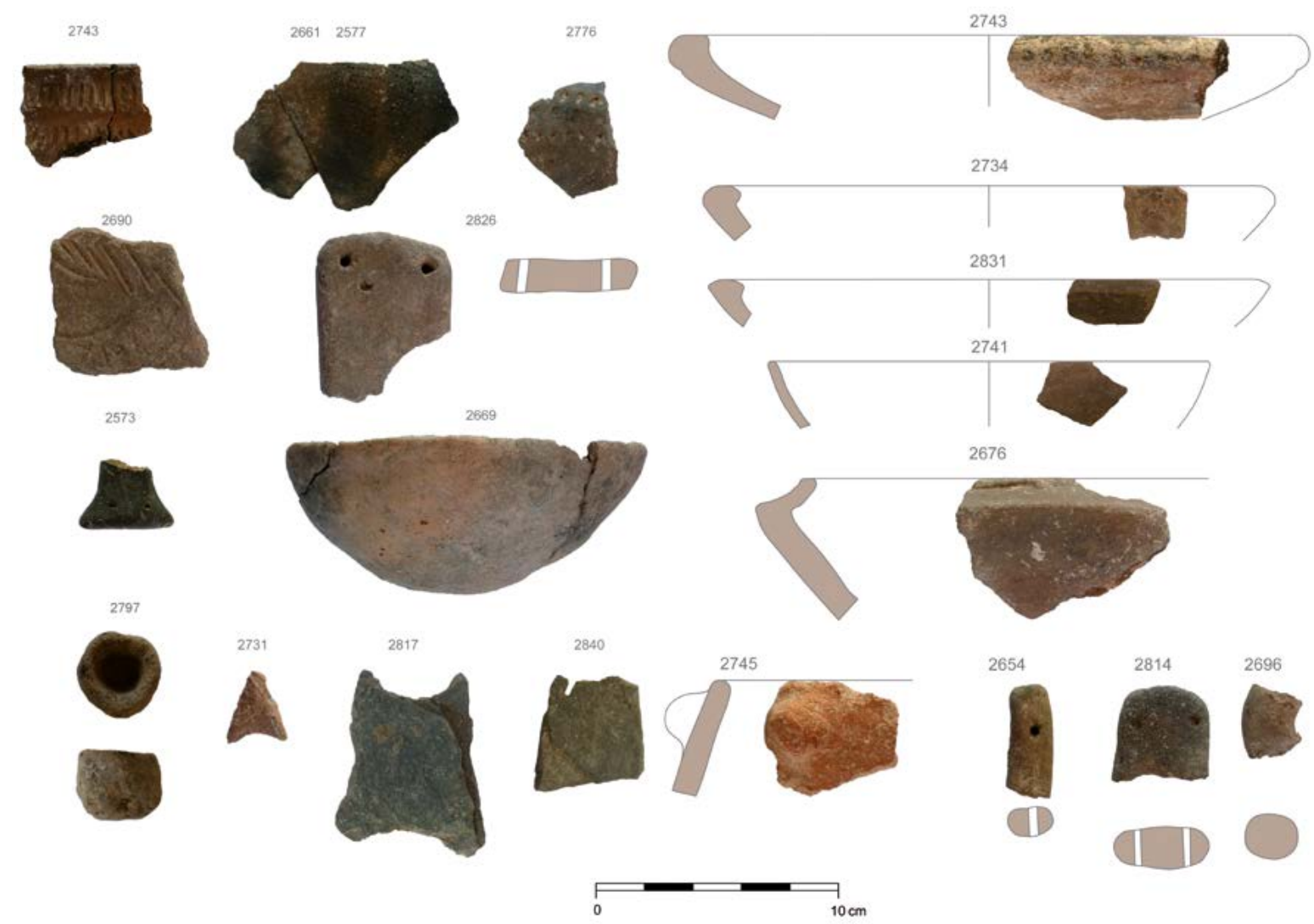

Fig. 8. Selección de materiales cerámicos y líticos $(2731,2817,2840)$ hallados en el relleno del Foso 2 de Perdigões (en color en la versión electrónica).

restos óseos que aparecen en los rellenos de este tipo de estructuras dificulta conocer el tiempo transcurrido entre la muerte del individuo y su depósito en el interior del foso. Además, la naturaleza eminentemente antrópica de los rellenos, la posibilidad de manipulación y traslados de restos orgánicos y la práctica de recortes del relleno con nuevas deposiciones cuyas diferencias cronológicas pueden ser importantes dificultan las condiciones necesarias para la datación de este tipo de estructuras.

Ante esta problemática y asumiendo dichas restricciones, los criterios de selección de las muestras han contemplado: 1) su buen estado de conservación y bajos niveles de meteorización; 2) garantizar su pertenencia a distintos individuos; 3) datar individuos diferentes de una misma unidad. Los objetivos específicos fueron: a) determinar si existen o no intervalos claros en la formación del relleno mediante la representación del mayor número posible de estratos; b) conocer el periodo de colmatación de la estructura estableciendo los límites temporales a los que corres- ponden las dataciones; c) verificar la coherencia interna de las dataciones respecto al orden estratigráfico o alternativamente detectar posibles discordancias.

Para ello se enviaron 15 muestras de fauna al laboratorio Beta Analytic. Doce de ellas proporcionaron resultados positivos, mientras que las tres restantes fueron descartadas por falta de colágeno. Fueron medidas por AMS (Accelerator Mass Spectrometry) y calibradas con la curva IntCal13 (Reimer et al. 2013), usando OxCal 4.3 (Bronk Ramsey 1995) (Tab. 1).

Las tres unidades cuyas muestras no pudieron ser datadas por falta de colágeno fueron las UU.EE 591, 581 y 547.

Durante los últimos años, la búsqueda de una mejor interpretación de los recintos de fosos europeos ha incorporado el estudio cronológico haciendo uso de la modelización bayesiana (Whittle et al. 2011; Aranda et al. 2016; Seidel et al. 2016; Balsera et al. 2015). Ello permite discutir la cronología de los fosos y sus rellenos desde nuevas perspectivas y con mayor número de dataciones para una misma estructura. 


\begin{tabular}{|c|c|c|c|c|c|c|c|c|c|c|c|}
\hline Contexto & Laboratorio & Método & $\begin{array}{c}\mathbf{N}^{\mathbf{0}} \mathbf{d e} \\
\text { muestra }\end{array}$ & Material & $\begin{array}{c}\text { Edad } \\
\text { BP }\end{array}$ & \pm & $\mathbf{C : N}$ & $\mathbf{\delta}^{15} \mathbf{N}$ & $\boldsymbol{\delta}^{13} \mathbf{C}$ & \multicolumn{3}{|c|}{ Calibración } \\
\hline UE605 & Beta & AMS & 461413 & $\begin{array}{c}\text { Mandíbula derecha } \\
\text { Sus } s p .\end{array}$ & 3940 & 30 & 3,3 & 5,8 & $-19,9$ & $2490-2350$ & $2570-2310$ \\
\hline UE602 & Beta & AMS & 461410 & $\begin{array}{c}\text { Ulna derecha } \\
\text { Sus sp. }\end{array}$ & 3820 & 30 & 3,3 & 5,8 & -20 & $2300-2200$ & $2450-2140$ \\
\hline UE603 & Beta & AMS & 461411 & $\begin{array}{c}\text { Mandíbula } \\
\text { Sus sp. }\end{array}$ & 3910 & 30 & 3,3 & 5 & $-20,1$ & $2470-2350$ & $2470-2300$ \\
\hline UE604 & Beta & AMS & 461412 & $\begin{array}{c}\text { Mandíbula } \\
\text { Sus sp. }\end{array}$ & 3830 & 30 & 3,3 & 4,8 & -20 & $2340-2210$ & $2460-2150$ \\
\hline UE596 & Beta & AMS & 461408 & $\begin{array}{c}\text { Mandíbula derecha } \\
\text { Sus sp. }\end{array}$ & 3840 & 30 & 3,3 & 4,2 & $-19,1$ & $2390-2210$ & $2460-2200$ \\
\hline UE585 & Beta & AMS & 461407 & $\begin{array}{c}\text { Mandíbula derecha } \\
\text { Canis familiaris }\end{array}$ & 3930 & 30 & 3,3 & 7,8 & $-19,1$ & $2470-2350$ & $2480-2300$ \\
\hline UE585 & Beta & AMS & 461406 & $\begin{array}{c}\text { Mandíbula derecha } \\
\text { Ovis/Capra }\end{array}$ & 3920 & 30 & 3,3 & 4,5 & 20,6 & $2480-2350$ & $2560-2300$ \\
\hline UE584 & Beta & AMS & 461405 & $\begin{array}{c}\text { Ulna derecha } \\
\text { Sus sp. }\end{array}$ & 3870 & 30 & 3,3 & 4,9 & $-19,6$ & $2450-2290$ & $2470-2210$ \\
\hline UE573 & Beta & AMS & 461403 & $\begin{array}{c}\text { Mandíbula derecha } \\
\text { Ovis/Capra }\end{array}$ & 3880 & 30 & 3.2 & 5,5 & $-19,7$ & $2460-2310$ & $2470-2240$ \\
\hline UE567 & Beta & AMS & 461402 & $\begin{array}{c}\text { Mandíbula derecha } \\
\text { Ovis/Capra }\end{array}$ & 3880 & 30 & 3.4 & 5,5 & $-20,6$ & $2460-2310$ & $2470-2240$ \\
\hline UE546 & Beta & AMS & 461400 & $\begin{array}{c}\text { Mandíbula derecha } \\
\text { Ovis/Capra }\end{array}$ & 3810 & 30 & 3.4 & 8,3 & $-19,8$ & $2290-2200$ & $2400-2140$ \\
\hline UE541 & Beta & AMS & 461399 & $\begin{array}{c}\text { Escápula izquierda } \\
\text { Sus sp. }\end{array}$ & 3800 & 30 & 3.3 & 4,7 & $-19,5$ & $2290-2200$ & $2340-2140$ \\
\hline
\end{tabular}

Tab. 1. Dataciones radiocarbónicas del Foso 2 en el sector L de Perdigões. Calibración con IntlCal13 (Reimer et al. 2013) (Oxcal 4.3). Todas las fechas calibradas han sido redondeadas a 10 años siguiendo a Stuiver y Polach (1977).

En el Foso 2 de Perdigões se llevó a cabo, en primer lugar, un test de contemporaneidad de las fechas disponibles, según el cual no correspondían a un único momento de deposición (T’29.2; T’ $(5 \%)=19.7$ $\mathrm{df}=11)($ Ward y Wilson 1978). En segundo lugar, un primer modelo bayesiano redujo los intervalos de probabilidad para lo que entendemos como conjunto de actividades de relleno de la estructura (Fig. 9). La ordenación cronológica sigue la posición estratigráfica de las muestras. Además, se consideraron dos fases claramente detectadas durante la excavación. Una corresponde a todas las actividades de colmatación del foso, es decir a su vida activa, y la otra a las acciones de época prehistórica efectuadas una vez amortizada esta estructura, momento al que pertenecen las dataciones más recientes (Beta 461400 y Beta 461399). Las fechas se redondearon a 5 años siguiendo la metodología de Bayliss et al. (2011) para contextos similares.

El modelo proporcionó un Aoverall de $74 \%$, con las siguientes fechas de inicio y fin para cada fase: la primera se encuadraría entre $2460-2365$ cal AC $(1 \sigma)$, o $2510-2320 \mathrm{cal} \mathrm{AC}(2 \sigma)$ y $2340-2270 \mathrm{cal}$ AC $(1 \sigma)$, o 2400-2230 cal AC (2 $\sigma)$, con un span o período de duración entre 0 y 180 años $(2 \sigma)$; la segunda se dispone entre 2340-2270 cal AC (1 $\sigma)$, o 2400-2230 cal AC $(2 \sigma)$ y $2285-2160$ cal AC $(1 \sigma)$, o $2340-1915$ cal $\mathrm{AC}(2 \sigma)$, con un span entre 0 y 90 años $(2 \sigma)$.

Este modelo es coherente, aunque presenta dos fechas con menor consistencia y, por tanto, inconsistentes con su posición estratigráfica (Beta 461410 y Beta 461412). Estas discordancias responderían a la naturaleza compleja del relleno, ya comentada.

Un segundo modelo considera el orden estratigráfico de las dataciones y los posibles cambios en las dinámicas internas de colmatación antes mencionadas (Fig. 10). En este segundo análisis bayesiano se han eliminado las dos fechas inconsistentes con la secuencia estratigráfica identificada. El modelo dio como resultado un Aoverall de $97 \%$, con las siguientes fechas de inicio y fin para cada fase: la primera dinámica se inicia en 2475-2350 cal AC ( 1 б), o 2490-2350 cal AC $(2 \sigma)$ y termina en $2460-2350 \mathrm{cal}$ AC $(1 \sigma)$, o 2470-2350 cal AC $(2 \sigma)$. Esta fase presenta un span o período entre 0 y 22 años $(2 \sigma)$. La segunda dinámica de colmatación se encuadra entre 2440-2345 cal AC 


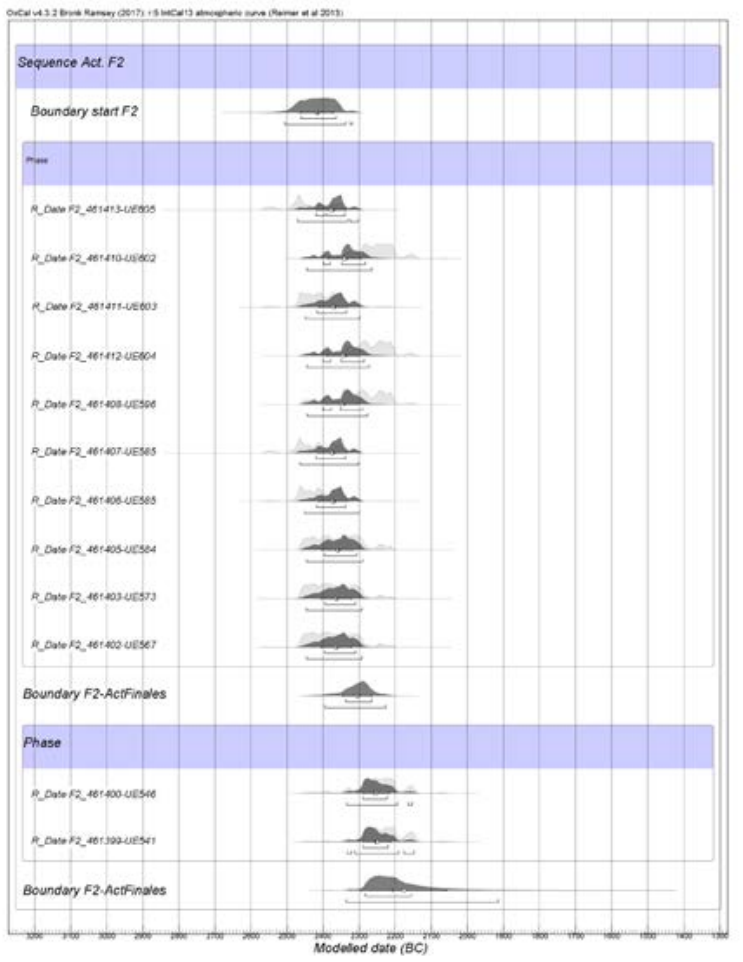

Fig. 9. Modelo bayesiano de las dataciones radiocarbónicas del Foso 2 de Perdigões. Las fechas se agrupan en dos fases diferenciando las acciones de relleno y las realizadas una vez colmatada la estructura. En gris claro se representan las dataciones radiocarbónicas y en gris oscuro los resultados del análisis bayesiano. Curva InCal 13 (Reimer et al. 2013) mediante el programa Oxcal 4.3.2 (Bronk Ramsey 2017).

$(1 \sigma)$, o $2460-2340$ cal AC $(2 \sigma)$ y $2410-2335$ cal AC $(1 \sigma)$, o $2460-2310$ cal AC $(2 \sigma)$, con un span de 0 y 42 años $(2 \sigma)$. La fase final, que responde a las actividades posteriores a la amortización de la estructura, se enmarca entre 2330-2240 cal AC ( 1 б), o 2380-2205 cal AC $(2 \sigma)$ y $2280-2190$ cal AC $(1 \sigma)$, o $2340-2080$ cal AC $(2 \sigma)$, con un span entre 0 y 71 años $(2 \sigma)$.

$\mathrm{La}$ interpretación de los dos modelos debe tener presente la desigual proporción de dataciones incluidas en cada fase, pero ambos muestran similitudes en los intervalos de probabilidades para las fechas de inicio y fin de las fases en las que se enmarcan las actividades en el interior del foso. En ambos casos, todos los estratos datados en el interior de la estructura se habrían formado entre 2500-2300 cal AC.

Los límites de los intervalos de probabilidades para el final de las actividades efectuadas sobre el relleno una vez amortizado el foso sí muestran diferencias entre los modelos. Sin embargo, estas diferencias no afectan al hecho de que, en ambos, las fechas finales son ligeramente posteriores al relleno, con medias centradas en el inicio del período 2250-2000 cal AC.

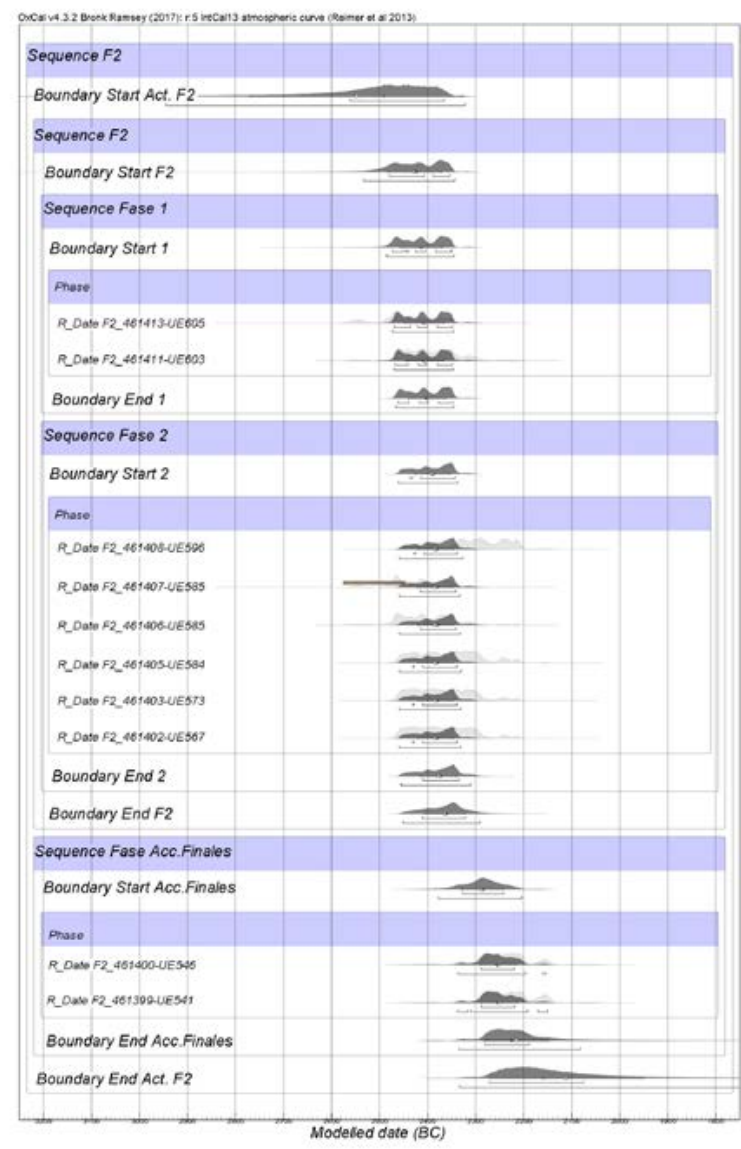

Fig. 10. Modelo bayesiano de las dataciones radiocarbónicas del Foso 2 de Perdigões. Las fechas se agrupan en tres fases diferenciando las dinámicas de relleno y las realizadas una vez colmatada la estructura. En gris claro se representan las dataciones radiocarbónicas y en gris oscuro los resultados del análisis bayesiano. Curva InCal 13 (Reimer et al. 2013) mediante el programa Oxcal 4.3.2 (Bronk Ramsey 2017).

El Foso 2 de Perdigões cuenta con 12 fechas absolutas y es el que más dataciones posee en todo el yacimiento. Ello no excluye que seamos conscientes de que nuestra interpretación está limitada por su número todavía reducido y su procedencia de un solo corte arqueológico, circunstancia generalizada, por otra parte, a toda la investigación de este tipo de yacimientos. Sin embargo, a la luz de los datos obtenidos resulta verosímil incluir el Foso 2, como se ha propuesto para el Foso 1, en lo que podríamos considerar como arquitectura monumental tardía del yacimiento. Ello les diferencia sensiblemente de los fosos centrales de Perdigões que han sido datados y discutidos por los principales investigadores de este yacimiento (Valera y Silva 2011; Valera 2013; Valera et al. 2014b, 2017). 


\section{RELACIÓN DE FOSO 1 Y FOSO 2}

Desde el momento de su descubrimiento, resultó evidente el paralelismo de los trazados de Foso 1 y Foso 2 de Perdigões. La regularidad mantenida a lo largo de todo su perímetro, los cinco accesos compartidos y las fosas estrechas (hasta 17) que se disponen radialmente entre los dos grandes fosos (Márquez et al. 2011b: 183), hacían pensar en dos obras gemelas o vinculadas. A esto se unía la forzada modificación del trazado de la zanja más externa (Foso 1) para configurar un espacio, a modo de gran bolsada semicircular (véase Fig. 2), donde se localizaban varias estructuras funerarias, algunas de ellas tipo tholos. En conjunto, la arquitectura periférica de Perdigões ofre- cía una curiosa fisonomía sin parangón en otros recintos peninsulares.

Esta circunstancia hizo que, desde un primer momento, un objetivo preferente de nuestros trabajos fuera comprobar si la evidente relación espacial de estos dos grandes fosos podía ser resultado de la contemporaneidad de ambas construcciones (Márquez et al. 2008: 29).

Cabe recordar que el Foso 1 (excavado entre 2008 y 2010) presenta una planta con aspecto de gran circunferencia de $470 \mathrm{~m}$ de diámetro y unos $1529 \mathrm{~m}$ de perímetro. Su sección en "V" tiene una anchura máxima documentada de $8,81 \mathrm{~m}$ y un mínimo de $2,37 \mathrm{~m}$ (en las proximidades de la puerta de acceso), mientras que su profundidad alcanza al menos los 3,38 $\mathrm{m}$ (Fig. 11).
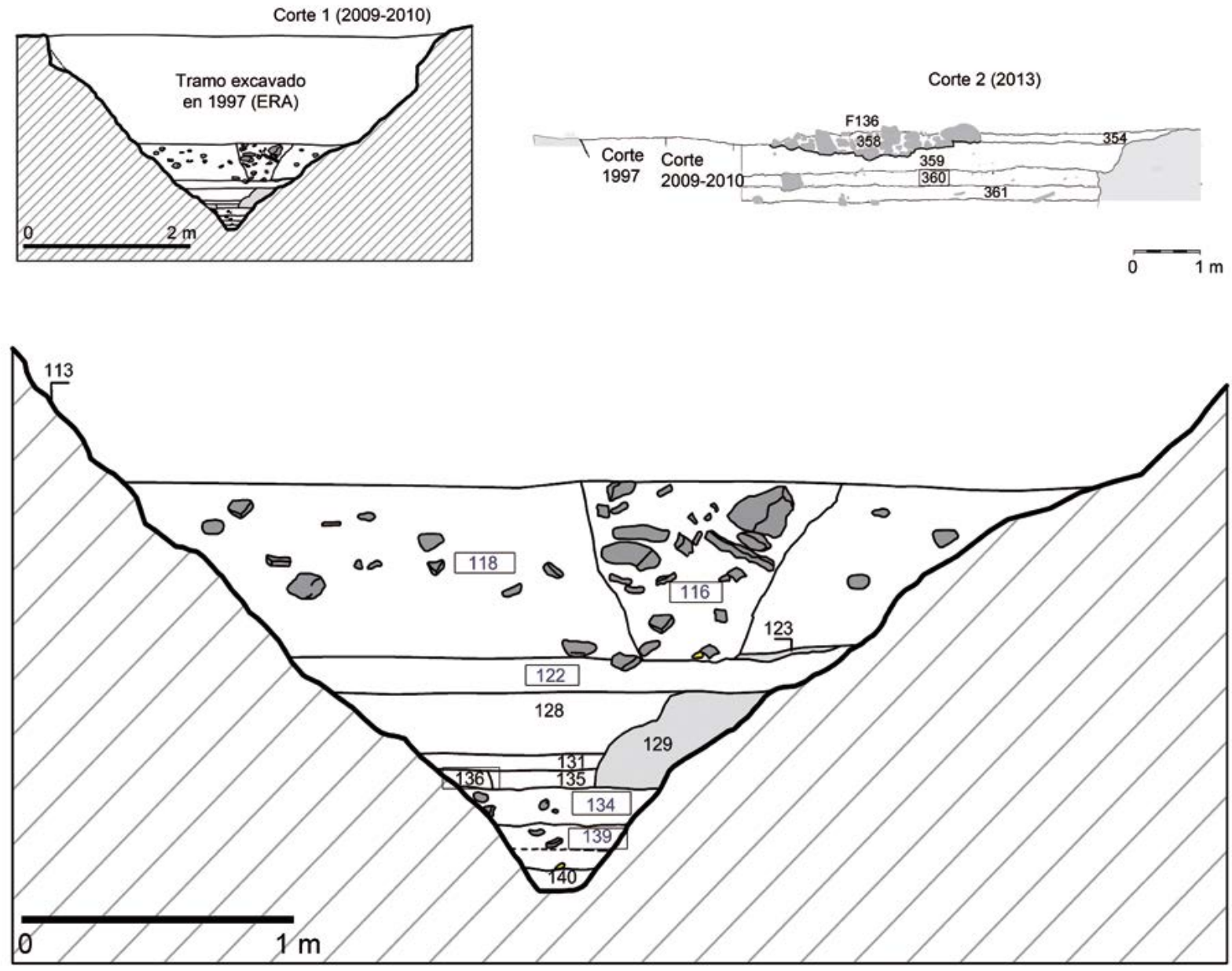

Detalle de secuencia estratigráfica excavada en Corte 1 (Universidad de Málaga)

Fig. 11. Perfil del Foso 1 de Perdigões con detalle de colmatación excavada por la Universidad de Málaga. Corte 1 y Corte 2 según se indica en Fig. 3. Las unidades estratigráficas datadas se recuadran. 
En su compleja estratigrafía interior (Márquez et al. 2013) se observan dos dinámicas de relleno distintas. La más profunda (UU.EE 139, 134, 133, 122), con cerca de $1 \mathrm{~m}$ de potencia, se caracteriza por unos estratos de naturaleza antrópica, conteniendo abundantes restos faunísticos y cerámicos, así como algo de industria lítica tallada y pulimentada. Sobre estos niveles se practicaron pequeñas fosas que, en algún caso se cortan unas con otras. Estos depósitos alternan a veces con otros de escasa potencia y estériles, de matriz semejante al sustrato geológico de base, previsiblemente de génesis coluvial. En un segundo momento (UU. EE. 118, 116, 360, 31, 11, 12), el Foso 1 se rellenó con estratos de mayor grosor y abundante material arqueológico, sin evidencia de fosas. Estos niveles superiores, interpretados como de génesis antrópica, superan en conjunto los $2 \mathrm{~m}$, y en ellos destaca la presencia de fragmentos de cerámica campaniforme incisa.

Para comparar la cronología absoluta de los Fosos 1 y 2 se ha realizado un nuevo análisis bayesiano que responde a la ordenación estratigráfica y a una sola fase que representa el conjunto de acciones de relleno, según el criterio del primer modelo elaborado para el Foso 2. Se han utilizado las 10 fechas del relleno de la estructura, ya discutidas en otras publicaciones (Márquez et al. 2013; Valera et al. 2014b; Caro et al.
2015; Caro 2017). Todas fueron obtenidas en el laboratorio Beta Analytic, medidas por AMS y calibradas con la curva IntCal13 (Reimer et al. 2013), usando OxCal (Bronk Ramsey 1995) en su versión 4.3 (Tab. 2). Dos fechas, poco consistentes, fueron incorporadas como outliers, tal y como se contempló en Márquez et al. (2013).

El modelo presenta un Aoverall de $92 \%$, propone el inicio de la fase entre 2400-2290 cal AC (1 б), o 2490-2235 cal AC $(2 \sigma)$ y el final de la colmatación

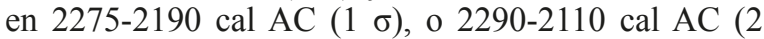
$\sigma)$, con un span de entre 0 y 340 años $(2 \sigma)$, para el total de las acciones de relleno que hemos registrado (Fig. 12).

Estos resultados son comparables con un modelo más complejo que recientemente hemos discutido (Caro 2017) donde reflejamos las dos dinámicas que conforman el relleno de esta estructura. Los resultados presentan fechas de inicio y fin entre 2410-2300 cal AC (1 $\sigma)$, o $2560-2280 \mathrm{cal} \mathrm{AC}(2 \sigma)$ y $2395-2220 \mathrm{cal} \mathrm{AC}(2$ $\sigma)$, o 2340-2265 cal AC $(1 \sigma)$, con un span de entre 0 y 161 años para la primera fase. La segunda fase tiene fechas entre 2340-2265 cal AC (1 $\sigma)$, o 2395-2220 cal AC $(2 \sigma)$ y $2290-2190$ cal AC $(1 \sigma)$, o $2330-2000 \mathrm{cal}$ $\mathrm{AC}(2 \sigma)$, con un span entre 0 y 196 años $(2 \sigma)$.

Ambos modelos señalan fechas de inicio y finalización que sitúan las actividades de relleno de Foso 1

\begin{tabular}{|c|c|c|c|c|c|c|c|}
\hline \multirow{2}{*}{ Contexto } & \multirow{2}{*}{ Laboratorio } & \multirow{2}{*}{$\mathbf{N}^{0}$ de muestra } & \multirow{2}{*}{ Material } & \multirow{2}{*}{ Edad BP } & \multirow{2}{*}{ \pm} & \multicolumn{2}{|c|}{ Calibración } \\
\hline & & & & & & $(68 \%)$ cal AC & $(95 \%)$ cal AC \\
\hline UE139a & Beta & 315725 & $\begin{array}{l}\text { Hueso de mamífero } \\
\text { de tamaño medio }\end{array}$ & 3890 & 30 & $2460-2350$ & $2470-2290$ \\
\hline UE134 & Beta & 315723 & $\begin{array}{c}\text { Hueso de mamífero } \\
\text { de tamaño medio }\end{array}$ & 3820 & 30 & $2300-2200$ & $2450-2140$ \\
\hline UE133 & Beta & 315722 & $\begin{array}{l}\text { Diente } \\
\text { Sus sp. }\end{array}$ & 3840 & 30 & $2460-2350$ & $2470-2290$ \\
\hline UE122 & Beta & 315721 & $\begin{array}{l}\text { Hueso de mamífero } \\
\text { de tamaño medio }\end{array}$ & 3840 & 30 & $2390-2210$ & $2460-2200$ \\
\hline UE118 & Beta & 315719 & $\begin{array}{c}\text { Diente } \\
\text { Ovis/Capra }\end{array}$ & 3780 & 30 & $2280-2140$ & $2300-2060$ \\
\hline UE116 & Beta & 315720 & $\begin{array}{c}\text { Diente } \\
\text { Ovis/Capra }\end{array}$ & 3860 & 30 & $2450-2290$ & $2460-2210$ \\
\hline UE360 & Beta & 374731 & $\begin{array}{c}\text { Hueso } \\
\text { Inclasificable }\end{array}$ & 3870 & 30 & $2450-2290$ & $2470-2210$ \\
\hline UE31 & Beta & 315718 & $\begin{array}{l}\text { Mandíbula } \\
\text { Sus sp. }\end{array}$ & 4060 & 30 & $2830-2500$ & $2840-2480$ \\
\hline UE11 & Beta & 315716 & $\begin{array}{l}\text { Húmero } \\
\text { Sus sp. }\end{array}$ & 3770 & 30 & $2280-2140$ & $2290-2050$ \\
\hline UE12 & Beta & 315717 & $\begin{array}{c}\text { Astrágalo } \\
\text { Cervus elaphus. }\end{array}$ & 3980 & 30 & $2570-2470$ & $2580-2460$ \\
\hline
\end{tabular}

Tab. 2. Dataciones radiocarbónicas del Foso 1 de Perdigões. Calibración con IntlCal13 (Reimer et al. 2013) (Oxcal 4.3). Todas las fechas calibradas han sido redondeadas a 10 años siguiendo a Stuiver y Polach (1977). 


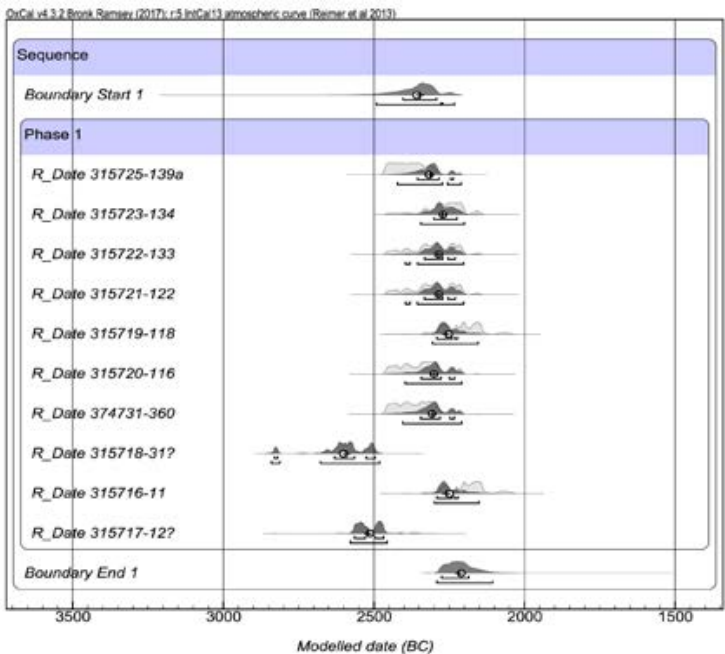

Fig. 12. Modelo bayesiano de las dataciones del Foso 1 de Perdigões. Las fechas se agrupan en una fase respetando el orden estratigráfico de las acciones de colmatación. En gris claro se representan las dataciones radiocarbónicas y en gris oscuro los resultados del análisis bayesiano. Curva InCal 13 (Reimer et al. 2013) mediante el programa Oxcal 4.3.2 (Bronk Ramsey 2017).

a lo largo del período comprendido entre 2500-2200cal AC. El segundo modelo enmarca la segunda dinámica de relleno en un momento levemente posterior a la primera fase, con medias centradas entre 2250-2000 cal $\mathrm{AC}$, aunque con una horquilla temporal amplia.

Tomando en cuenta la información cronológica de ambos fosos es posible establecer que su construcción y la mayoría de las actividades de sus rellenos se sitúa entre 2500-2250 cal AC. Esto nos lleva a afirmar que, por el momento, tal y como se ha avanzado, los rellenos de estos fosos son los más tardíos del yacimiento.

Además se observan dinámicas de relleno semejantes entre ambas estructuras, donde predominan los depósitos de naturaleza antrópica con alternancia de episodios ocasionales de naturaleza coluvial. También comparten pequeñas fosas $u$ hoyos excavados sobre los propios niveles de colmatación. Por el contrario, existen algunas diferencias. Por ejemplo, en el episodio final de relleno del Foso 1, correspondiente a su definitiva amortización, había evidencias de potentes rellenos antrópicos realizados desde el exterior y ausencia de pequeñas fosas, mientras que en el Foso 2 los estratos tienden a la horizontalidad y su amortización final parece ser más pausada. Tampoco hemos encontrado evidencia alguna de grandes recortes (recuttings) en el Foso 2 mientras que en su vecino gemelo se practicó uno en sentido longitudinal, con colmatación de piedras, cuando la estructura estaba totalmente colmatada (véase perfil en Fig. 11).

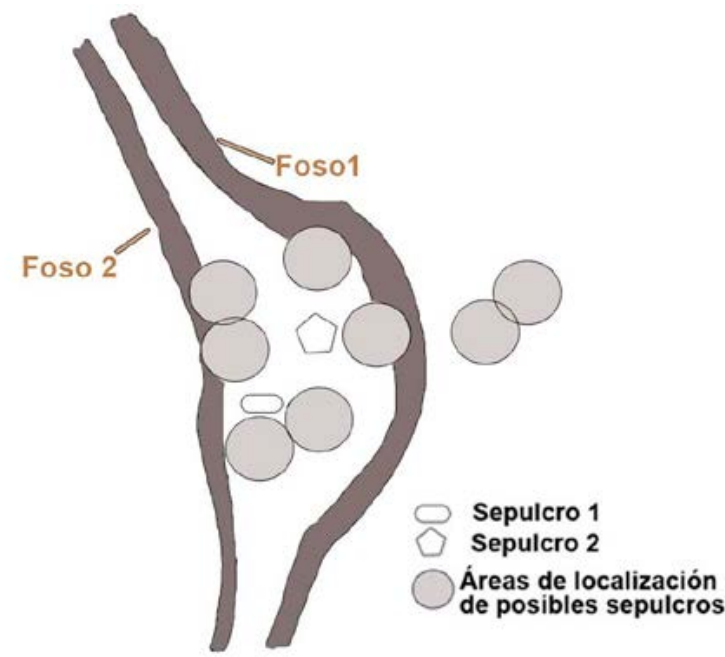

Fig. 13. Representación de la zona funeraria entre el Foso 1 y el Foso 2 de Perdigões (a partir de Valera et al. 2000).

Cabe señalar, en la cultura material localizada en el relleno de ambas estructuras, algunos patrones comunes que también se observan en otras grandes estructuras de Perdigões según la información disponible. Son la marcada fragmentación de los objetos cerámicos, el predominio de las formas abiertas y de grandes dimensiones (en especial fuentes de borde engrosado), el alto porcentaje de restos faunísticos y el escaso número de industria lítica tallada. Todos estos rasgos señalan similares conductas sociales de consumo y tratamiento de los objetos, y por tanto códigos culturales compartidos entre quienes participaron en la construcción de ambos fosos y de sus correspondientes rellenos.

Las dataciones y dinámicas de colmatación observadas en las dos estructuras permiten considerar diversos "modelos biográficos", al menos en este sector correspondiente a la Puerta 1 . Una propuesta sería que el Foso 2, al parecer algo más antiguo, pudo estar activo y amortizado antes de la construcción de Foso 1. Otra propondría que, antes de ser colmatado el Foso 2, tal vez a lo largo de su segunda fase de relleno, se excavase el Foso 1 y se iniciasen los vertidos en el mismo, pudiendo haber llegado a coincidir en el tiempo las actividades de relleno de ambos fosos.

\section{RECINTOS EXTERIORES Y SU RELACIÓN CON LOS SEPULCROS MEGALÍTICOS TIPO THOLOS}

Los restos humanos que se han hallado en diferentes estructuras negativas excavadas en el yacimiento 
son numerosos (Valera y Godinho 2010; Valera et al. 2014a). Sin embargo desde el inicio de las investigaciones se conocía, al menos, un espacio funerario, definido por varias estructuras que ocupaban la gran bolsada semicircular existente entre los dos fosos más exteriores del yacimiento (Valera et al. 2000: 89).

Las oportunas excavaciones realizadas en el lugar y posteriores estudios antropológicos (Valera et al. 2000; Evangelista y Silva 2013; Valera et al. 2014a; Silva et al. 2017) confirmaron la existencia de dos estructuras tipo tholos (Fig. 13) y localizaron otras estructuras en el entorno tal y como se refleja en la figura. Estas últimas están pendientes de caracterizar, a excepción de la llamada "tumba III" que se ha identificado como de tipo fosa, aunque junto con las anteriores, al carecer de dataciones conocidas no puede incorporarse a nuestra discusión. De hecho, las únicas dataciones radicarbónicas conocidas corresponden a los dos sepulcros megalíticos (Valera et al. 2014b).

El primer tholos (sepulcro 1) es una estructura semi-subterránea, excavada en la roca, que se localiza en el centro del espacio funerario y tiene una orientación E-O. Presenta en su extremo occidental una cámara de forma circular de 3,5 $\mathrm{m}$ de diámetro, y al este un corredor de $1,8 \mathrm{~m}$ de largo, al que sigue el denominado atrio semicircular de $2 \mathrm{~m}$ de diámetro. Según los estudios publicados, el número mínimo de individuos (NMI) depositados en su interior alcanzaría los 106 (Valera et al. 2014a). Entre los elementos de ajuar hay cuentas de collar, conchas, placas de esquisto, ídolos falange, un recipiente realizado en hueso, alabardas, vasos cerámicos y de piedra, láminas y una alabarda en sílex, así como objetos exógenos en variscita o marfil. En el interior se ve el derrumbe de las losas que estaban dispuestas en las paredes, así como evidencias de diferentes fases de utilización, previas y posteriores a dicho episodio. Las dataciones (Tab. 3) se han realizado sobre cuatro restos humanos (Valera et al. 2014a: 40-42, 2014b: 15). Los localizados en estratos previos al derrumbe han aportado las fechas $2840-2470$ y $2580-2470$ cal AC (2 $\sigma)$ (Beta-327750 y Beta-311480, respectivamente) y los restos posteriores al mismo las fechas $2840-2480$ y $2870-2580$ cal AC $(2 \sigma)$ (Beta-327748 y Beta-327747).

El sepulcro 2 se localiza unos $20 \mathrm{~m}$ al noreste de la anterior. También está parcialmente excavada en la roca y se orienta al sureste. La cámara de planta circular mide $3 \mathrm{~m}$ de diámetro. Le sigue un corredor estrecho de $1 \mathrm{~m}$ de largo y un atrio elipsoidal. El NMI conocido para los restos hallados en su interior es de 26 en el atrio y 30 en la cámara (Silva et al. 2017). Entre los elementos de ajuar se identifican fragmentos cerámicos, puntas, objetos en hueso y marfil, botones en hueso, figuras zoomórficas, ídolos y láminas de oro (Valera et al. 2000; Schuhmacher y Banerjee 2012; Silva et al. 2017).

La construcción cuenta con cuatro dataciones asociadas a las diferentes fases de uso (Tab. 3) que se evidenciaron en su excavación (Valera et al. 2014a: 40-42, 2014b: 15). El inicio de los depósitos parecía restringirse a la cámara, con fecha 2860-2500 cal AC $(2 \sigma)$ (Beta-308791). Posteriormente esta sería parcialmente vaciada para una reutilización del sepulcro con fecha 2470-2290 cal AC (Beta-308792), del atrio con fechas $2580-2350$ cal AC y $2460-2200$ cal AC $(2 \sigma)$ (Beta- 308789 y Beta-308793) y del corredor.

El número de dataciones de cada sepulcro es escaso en comparación con los fosos, pero las fechas más antiguas en ambas estructuras indican un primer uso centrado entre 3000-2500 cal AC y, las siguientes, una reutilización durante el período 2500-2250 cal AC, coincidente con el momento en que se están colmatando los Fosos 1 y 2.

Como ya se ha apuntado, los datos conocidos durante los primeros años de investigación hacían pensar que la llamada "necrópolis" se había construido en un área seleccionada y demarcada ex profeso por el en-

\begin{tabular}{|c|c|c|c|c|c|c|c|}
\hline \multirow{2}{*}{ Contexto } & \multirow{2}{*}{ Laboratorio } & \multirow{2}{*}{$\begin{array}{c}\mathrm{N}^{0} \text { de } \\
\text { muestra }\end{array}$} & \multirow{2}{*}{ Material } & \multirow{2}{*}{ Edad BP } & \multirow{2}{*}{ \pm} & \multicolumn{2}{|c|}{ Calibración } \\
\hline & & & & & & $(68 \%)$ cal AC & $(95 \%)$ cal AC \\
\hline Tholos 1_173 & Beta & 327750 & Hueso humano & 4030 & 40 & $2580-2480$ & $2840-2470$ \\
\hline Tholos 1 & Beta & 311480 & Hueso humano & 3990 & 30 & $2570-2470$ & $2580-2470$ \\
\hline Tholos 1_93 & Beta & 327748 & Hueso humano & 4060 & 30 & $2830-2500$ & $2840-2480$ \\
\hline Tholos 1_84 & Beta & 327747 & Hueso humano & 4130 & 30 & $2860-2630$ & $2870-2580$ \\
\hline Tholos 2_458 (inicial) & Beta & 308791 & Hueso humano & 4090 & 30 & $2840-2580$ & $2860-2500$ \\
\hline Tholos 2_429 & Beta & 308792 & Hueso humano & 3890 & 30 & $2460-2350$ & $2470-2290$ \\
\hline Tholos 2_231 & Beta & 308793 & Hueso humano & 3970 & 30 & $2560-2470$ & $2580-2350$ \\
\hline Tholos 2_232 & Beta & 308789 & Hueso humano & 3840 & 30 & $2390-2210$ & $2460-2200$ \\
\hline
\end{tabular}

Tab. 3. Dataciones radiocarbónicas de los tholoi 1 y 2 de Perdigões (a partir de Valera et al. 2014a, 2014b). Calibración con IntlCal13 (Reimer et al. 2013) (Oxcal 4.3.2). Todas las fechas calibradas han sido redondeadas a 10 años siguiendo a Stuiver y Polach (1977). 
sanchamiento del perímetro del foso externo (Valera et al. 2000: 88). Sin embargo, un mejor conocimiento de la biografía del yacimiento, especialmente el estudio pormenorizado de la cronología de Foso 1 (Márquez et al. 2013: 27; Valera et al. 2014b) y Foso 2, nos permite reinterpretar las relaciones espaciales y cronológicas entre todas estas construcciones.

La suma de probabilidades (Fig. 14) que sigue nos sirve para proponer su ubicación temporal en el conjunto del yacimiento de forma orientativa y a la luz de las dataciones radiocarbónicas conocidas. Tenemos en cuenta las importantes limitaciones de este recurso, que ya vienen siendo apuntadas por los especialistas en la materia (p. ej. Bayliss et al. 2007; Michczyński y Michczyńska 2006; Chiverrel et al. 2011; Contreras y Meadows 2014) ${ }^{3}$. En este sentido, advertimos que la cantidad de dataciones por estructuras es desigual, siendo los Fosos 1 y 2 los que cuentan con un número marcadamente superior. En todo caso, el gráfico nos permite identificar tendencias que vendrían a reafirmar nuestro anterior análisis.

De acuerdo con los resultados, el primer uso de los sepulcros tipo tholos sería previo a los Fosos 1 y 2, así como el del resto de los fosos. Las dos mayores

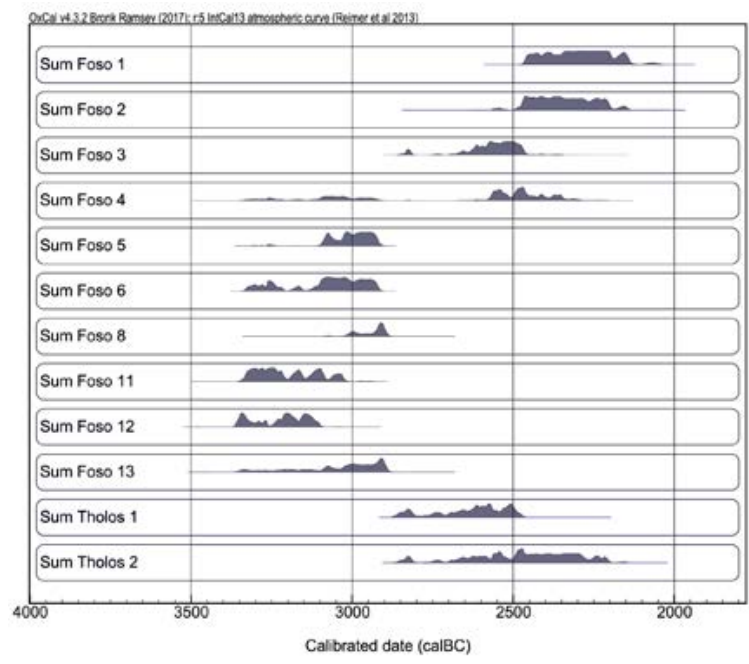

Fig. 14. Distribuciones de sumas de probabilidades de los Fosos 1, $2,3,4,5,6,8,11,12,13$ y tholoi 1 y 2 de Perdigões (a partir de Márquez et al. 2013; Valera et al. 2014a, 2014b, 2017 y dataciones en esta publicación). Curva InCal 13 (Reimer et al. 2013) mediante el programa Oxcal 4.3.2 (Bronk Ramsey 2017).

\footnotetext{
${ }^{3}$ Los resultados de esta herramienta metodológica están afectados por la calibración, la desviación típica de las dataciones y, muy en especial, por el número de fechas que se suman para cada actividad, ya que cuantas más fechas haya, más larga será la estimación de la duración de las actividades.
}

estructuras construidas en el lugar corresponden a una etapa de arquitectura monumental tardía dentro de momentos avanzados de la Edad del Cobre.

Con esta nueva información cabe pensar que las viejas construcciones funerarias se incorporaron, sin contradicción alguna, en las fases finales del yacimiento, quizá dentro ya de nuevas claves ontológicas o culturales pero como elementos con marcada personalidad arquitectónica y nuevos usos. La modificación en el trazado de Foso 1 es notoria. En este nuevo contexto tendrían sentido las evidencias de reutilización detectadas en el sepulcro 2, ya en momentos entre 2500-2000 cal AC (Valera et al. 2014b: 21), contemporáneos, en tal caso, con la actividades de relleno registrada en los dos grandes fosos. Estaríamos previsiblemente ante una dinámica de apropiación simbólica de elementos del pasado, bien constatada por la arqueología en otros tantos recintos de fosos europeos, manifestada por las frecuentes reutilizaciones de viejos sepulcros o mediante la práctica de recortes y rellenos de antiguas estructuras negativas (Márquez Romero y Jiménez Jáimez 2010).

\section{CONCLUSIÓN}

Los nuevos resultados aportados por la investigación del Foso 2 de Perdigões han posibilitado disponer de 12 nuevas dataciones radiocarbónicas inéditas que, junto a la serie de 10 fechas del Foso 1, nos permiten identificar una fase tardía en la arquitectura monumental de dicho yacimiento. Esta fase ocupa su límite espacial exterior y presenta las estructuras de mayores dimensiones constructivas de todas las conocidas. Esta fase se centra entre $2500-2250 \mathrm{cal} \mathrm{AC}$, coincidiendo con lo que se ha propuesto como la fase final de carácter monumental del fenómeno de los recintos de fosos del sur de la península ibérica (Márquez Romero y Jiménez Jáimez 2013: 455).

Además, se confirman dinámicas sociales donde la continua superposición de actividades, la reelaboración de la arquitectura del lugar y el uso del espacio permiten estrategias tanto de invisibilización de elementos del pasado, como ocurre con la Zanja 14 eliminada por la construcción del Foso 2, como la inclusión de otros elementos más antiguos en el trazado arquitectónico, caso de los sepulcros de falsa cúpula. Todo nos habla de unos importantes procesos de apropiación ideológica, que la arquitectura monumental con su persistencia en el paisaje siempre ha favorecido.

Los estudios realizados nos advierten de la compleja realidad arqueológica a la que nos enfrentamos en un tipo de yacimientos en los que se produce una continua superposición de eventos, lo que desaconseja aplicar criterios cartesianos de proximidad entre las estructuras para analizar su temporalidad. A la vez,

Trab. Prehist., 76, N. ${ }^{\circ}$ 1, enero-junio 2019, pp. 161-176, ISSN: 0082-5638

https://doi.org/10.3989/tp.2019.12232 
pone en evidencia la necesaria contrastación de información mediante la investigación arqueológica sistemática para comprender su dilatada historia.

\section{AGRADECIMIENTOS}

A Gonzalo Aranda Jiménez por sus sugerencias, a Francisco Melero, Antonio Oliver y Rodrigo Álvarez por su colaboración en las excavaciones y a Tamara Santiago en las tareas de laboratorio.

\section{BIBLIOGRAFÍA}

Aranda Jiménez, G.; Lozano Medina, A.; Escudero Carrillo, J.; Sánchez Romero, M.; Alarcón García, E.; Fernández Martín, S.... y Barba Colmenero, V. 2016: "Cronología y temporalidad de los recintos de fosos prehistóricos: el caso de Marroquíes Bajos (Jaén)". Trabajos de Prehistoria 73 (2): 231-250.

Balsera, V.; Bernabeu Aubán, J.; Costa-Caramé, M.; Díaz-del-Río, P.; García Sanjuán y Pardo, S. 2015: "The Radiocarbon Chronology of Southern Spain's Late Prehistory (5600 - 1000 cal BC): a comparative review". Oxford Journal of Archaeology 34 (2): 139-156.

Bayliss, A.; Bronk Ramsey, C.; Van Der Plicht, J. y Whittle, A. 2007: "Bradshaw and Bayes: towards a timetable for the Neolithic". Cambridge Archaeological Journal 17 (1 suppl.): 1-28.

Bayliss, A.; van der Plicht, J.; Bronk Ramsey; McCormac, G.; Healy, F. y Whittle, A. 2011: "Towards generational time-scales: the quantitative interpretation of archaeological chronologies". En A. Whittle, F. Healy y A. Bayliss: Gathering Time. Dating the Early Neolithic Enclosures of Southern Britain and Ireland 1. Oxbow books. Oxford: 17-59.

Bronk Ramsey, C. 1995: "Radiocarbon calibration and analysis of strtigraphy: the Oxcal program". Radiocarbon 37 (2): 42-430.

Caro, J. L. 2017: "Problemática para la datación en recintos de fosos usando análisis bayesiano: Complejo Arqueológico de Perdigões (Portugal) y la puerta 1 como caso de estudio". En J. A. Barceló, I. Bogdanovic y B. Morell (eds.): IberCrono. Cronometrías para la Historia de la Península Ibérica. Actas del Congreso de Cronometrías para la Historia de la Península Ibérica (IberCrono 2017, Barcelona 2016). CEUR-Workshop Proceedings 2024 urn:nbn:de:0074-2024-4.

Caro, J. L.; Compaña, J. M.; Rodríguez-Vinceiro, F. J. y Santamaría, J. A. 2015: "Primeros vestigios de metalurgia en el foso 1 (Puerta 1) del yacimiento de Perdigões. Un avance arqueométrico (Reguengos de Monsaraz, Portugal)". En N. Medina Rosales (ed.): VII Encuentro de Arqueología del suroeste peninsular. Aroche (España)/ Serpa (Portugal) (Aroche 2013): 279-298. Aroche.

Chiverrell, R. C.; Thorndycraft, V. R. y Hoffman, T. 2011: "Cumulative probability functions and their role in evaluating the chronology of geomorphological events during the Holocene". Journal of Quaternary Science 26 (1): 76-85.

Contreras, D. A. y Meadows, J. 2014: "Summed radiocarbon calibrations as a population proxy: a critical evaluation using a realistic simulation approach". Journal of Archaeological Science 52: 591-608.

Evangelista, L. S y Silva, A. M. 2013: “Tomb 3-Perdigões prehistoric enclosure (Reguengos de Monsaraz, Portugal): first anthropological results". Apontamentos de Arqueologia e Património 9: 47-54.

Lago, M.; Duarte, C.; Valera, A.; Albergaria, J.; Almeida, F. y Carvalho, A. 1998: "Povoado dos Perdigões (Reguengos de Mosaraz): dados preliminares dos trabalhos arqueológicos realizados em 1997'. Revista Portuguesa de Arqueologia 1 (1): 45-152.

Márquez, J. E.; Jiménez, V. y Mata, E. 2008: "Excavaciones en el yacimiento de Perdigões (Reguengos de Monsaraz, 2008-2010). Universidad de Málaga (España)". Apontamentos de Arqueologia e Património 2: 41-48.
Márquez Romero, J. E. y Jiménez Jáimez, V. 2010: Recintos de fosos Genealogía y significado de una tradición en la Prehistoria del suroeste de la Península Ibérica (IV-III milenios AC). Servicio de Publicaciones e Intercambio Científico, Universidad de Málaga. Málaga.

Márquez Romero, J. E. y Jiménez Jáimez, V. 2013: “Monumental ditched enclosures in southern Iberia (fourth-third millenia BC)". Antiquity 87: 447-460.

Márquez Romero, J. E.; Mata, E.; Jiménez Jáimez, V. y Suárez, J. 2013: "Dataciones absolutas para el Foso 1 de Perdigões (Reguengos de Monsaraz, Portugal). Reflexiones sobre su cronología y temporalidad". Spal 22: 17-27.

Márquez Romero, J. E.; Suárez Padilla, J.; Jiménez Jáimez, V. y Mata Vivar, E. 2011a: "Avance a la secuencia estratigráfica del "foso 1" de Perdigōes (Reguengos de Monsaraz, Portugal) a partir de las campañas 2009 y 2010". Menga. Revista de Prehistoria de Andalucía 2: $157-175$.

Márquez Romero, J. E.; Valera, A. C.; Becker, H.; Jiménez Jáimez, V. y Suárez Padilla, J. 2011b: "El complexo arqueológico dos Perdigōes (Reguengos de Monsaraz, Portugal). Prospecciones geofísicas - campañas 2008-2009". Trabajos de Prehistoria 68 (1): 175-186.

Michczyński, A. y Michczyńska, D. J. 2006: "The effect of PDF peaks' height increase during calibration of radiocarbon date sets". Geochronometria 25: 1-4.

Reimer, P. J.; Bard, E.; Bayliss, A.; Beck, J. W.; Blackwell, P. G.; Bronk Ramsey, C... y Van der Plicht, J. 2013: "IntCal13 and Marine13 Radiocarbon Age Calibration Curves 0-50,000 Years cal BP". $R a$ diocarbon 55 (4): 1869-1887.

Schuhmacher, T. X. y Banerjee, A. 2012: "Procedencia e intercambio de marfil en el Calcolítico de la Península Ibérica". En Actas del Congrés Internacional Xarxes al Neolític, Gavà-Barcelona. Rubricantum. Revista del Museo de Gavà 5. Gavà: 289-298.

Seidel, U.; Stephan, E.; Stika, H. P.; Dunbar, E.; Kromer, B.; Bayliss, A... y Whittle, A. 2016: "Die Zeit der groben Gräben: Modelle zur Chronologie des Michelsberger Fundplatzes von Heilbronn-Klingenberg 'Schlossberg', Stadkreis Heilbronn, Baden-Württemberg”. Praehistorische Zeitschrift 91 (2): 225-283. https://doi.org/10.1515/pz-2016-0022

Silva, A. M; García, M.; Leandro, I.; Evangelista, L. S.: Rodrigues, T. y Valera, A. C. 2017: "Mortuary practices in Perdigoes (Reguegos de Monsaraz, Portugal): bio-anthropological approach to tomb 2". Menga. Revista de Prehistoria de Andalucia 8: 71-86.

Suárez-Padilla, J.; Caro Herrero, L.; Mata Vivar, E.; Márquez Romero, J. E. y Jiménez Jáimez, V. 2013: "Excavaciones en extensión de la Universidad de Málaga (UMA) en el yacimiento de Perdigões (Reguengos de Monsaraz, Portugal). El sondeo L1. Campañas 2011-2012”. En J. Jiménez Avila, M. Bustamante-Álvarez y M. García Cabezas (coords.): VI Encuentro de Arqueología del Suroeste Peninsular (Villafranca de los Barros 2012): 521-550. Villafranca de los Barros.

Suárez-Padilla, J.; Márquez Romero, J. E.; Caro Herrero, J. L.; Mata Vivar, E.; Cuevas Albadalejo, P.; Jiménez Jaimez, V.... y CrespoLópez, E. 2015: "Excavaciones arqueológicas en la Puerta 1 del yacimiento de Perdigões (Reguengos de Monsaraz, Portugal). Universidad de Málaga. Campaña de 2013”. En N. Medina Rosales (ed.): VII Encuentro de Arqueología del suroeste peninsular. Aroche (España)/ Serpa (Portugal) (Aroche 2013): 279-298. Aroche.

Stuiver, M. A. y Polach, H. A. 1977: "Reporting the rate of 14C data". Radiocarbon 19: 355-363.

Valera, A. C. 2013: "Cronología dos recintos de fossos da pré-historia recente em território português". En J. Morais Arnaud, A. Martins, C. Neves (eds.): Arqueologia em Portugal 150 anos. Associaçao dos Arqueólogos Portugueses. Oficina de Artes Gráficas. Lisboa.

Valera, A. C. 2017: "Duas décadas de investigação nos Perdigões: resenha da bibliografia produzida". Apontamentos de Arqueología e Património 12: 59-75.

Valera, A. C. y Godinho, R. M. 2010 "Ossos humanos provenientes dos fossos 3 e 4 e gestão da morte nos Perdigões". Apontamentos de Arqueologia e Património 6: 29-39.

Valera, A. C.; Lago, M.; Duarte, C. E. y Evangelista, L. 2000: “Ambientes funerários no complexo arqueológico dos Perdigões: uma 
análise preliminar no contexto das práticas funerárias calcolíticas no Alentejo". ERA-Aqueologia 2: 84-105.

Valera, A. C. y Silva, A. M. 2011: "Datações de radiocarbono para os Perdigões (1): contextos com restos humanos nos sectores I \& Q". Apontamentos de Arqueologia e Património 7: 7-14.

Valera, A. C.; Silva, A. M.; Cunha, C. y Evangelista, L. S. 2014a "Funerary practices and body manipulation at Neolithic and chalcolithic Perdigões ditched enclosures (South Portugal)". En A. Valera (ed.): Recent prehistoric enclosures and funerary practices in Europe. Proceedings of the International Meeting held at the Gulbenkian Foundation (Lisbon, Portugal 2012). British Archaeological Reports International Series 2676. Archaeopress. Oxford: 37-57.

Valera, A. C.; Silva, A. M. y Márquez-Romero, J. E. 2014b: "The temporality of Perdigões enclosures: absolute chronology of the structures and social practices". Spal 23: 11-26.
Valera, A. C.; Simão, I.; Nunes, T.; Do Pereiro, T. y Costa, C. 2017: "Neolithic ditched enclosures in southern Portugal (4th millennium bc): new data and new perspectives". Estudos do Quaternário 17: 57-76.

Varela Gomes, M. 1994: "Menires e cromeleques no complexo cultural megalítico portugués-trabalhos recentes e estado da questão". En Actas do seminario 'O megalitismo no Centro de Portugal' (Viseu 1994): 317-342. Viseu.

Ward, G. K. y Wilson, S. R. 1978: "Procedures for comparing and combining radiocarbon age determinations: a critique". Archaeometry 20: 19-31.

Whittle, A.; Healy, F. y Bayliss, A. 2011: Gathering Time. Dating the Early Neolithic Enclosures of Southern Britain and Ireland. Oxbow books. Oxford. 